University of Wollongong

Research Online

Faculty of Engineering - Papers (Archive)

Faculty of Engineering and Information

Sciences

$1-1-2012$

\title{
Effect of grain size on the hydrogen diffusion process in steel using cellular automaton approach
}

Druce P. Dunne

University of Wollongong, druce@uow.edu.au

E V. Pereloma

University of Wollongong, elenap@uow.edu.au

Nima Yazdipour

University of Wollongong, nimayaz@uow.edu.au

Follow this and additional works at: https://ro.uow.edu.au/engpapers

Part of the Engineering Commons

https://ro.uow.edu.au/engpapers/4326

\section{Recommended Citation}

Dunne, Druce P.; Pereloma, E V.; and Yazdipour, Nima: Effect of grain size on the hydrogen diffusion process in steel using cellular automaton approach 2012, 1568-1573.

https://ro.uow.edu.au/engpapers/4326

Research Online is the open access institutional repository for the University of Wollongong. For further information contact the UOW Library: research-pubs@uow.edu.au 


\title{
Effect of Grain Size on the Hydrogen Diffusion Process in Steel Using Cellular Automaton Approach
}

\author{
Nima Yazdipour ${ }^{1, \mathrm{a}}$, Druce Dunne ${ }^{1, \mathrm{~b}}$, Elena Pereloma ${ }^{1, \mathrm{c}}$ \\ ${ }^{1}$ Faculty of Engineering, University of Wollongong, Wollongong, NSW, 2522, Australia \\ animayaz@uow.edu.au, bdruce@uow.edu.au, celenap@uow.edu.au
}

Keywords: Hydrogen diffusion, Hydrogen embrittlement, Grain size, Cellular Automata.

\begin{abstract}
The role of microstructure in susceptibility to hydrogen uptake and property degradation is being evaluated using a number of high strength pipeline steels. To do so, a cellular automaton (CA) model has been used to examine the effect of grain size, as a first step in assessing the influence of microstructure. The simulation results of hydrogen diffusion into microstructures with different grain sizes are presented.
\end{abstract}

\section{Introduction}

A critical factor influencing the economic viability of hydrogen distribution is the potential to use or build on synergies with the existing energy infrastructure. The existing natural gas pipeline network is the logical infrastructure that could be utilised to accelerate the uptake of new hydrogen technologies. However, the deleterious effect of hydrogen on the physical and mechanical properties of the pipeline steels needs to be carefully investigated and predicted to ensure safe and reliable pipeline operation under hydrogen pressures in both static and cyclic loading conditions. The influence of hydrogen on the properties of current pipeline steels depends on the kinetics of absorption, the diffusion rate and the complex interaction of hydrogen with microstructural features present in the steel.

The main task of computational mathematics is to study the spatial dynamics of a physical medium [1]. However, two alternative approaches have been proposed to solve the problem: traditional partial differential equations and cellular automaton (CA). The kernel of a CA model is the fact that both time and space are initially given in a discrete form [1].

In view of such applications, it is important to have a reliable CA model of diffusion. It is not sufficient to have a rule which simulates qualitatively a diffusive behaviour. A good quantitative agreement with experiments is also needed. In particular, the artefacts of the model such as the discrete space and time should be irrelevant in the simulation. CA approaches to diffusion have already been proposed by several authors [2-6].

The Cellular Automaton (CA) technique has been used to predict the property degradation of microstructures with various properties (e.g. different grain size) under a given hydrogen embrittlement condition. Very few studies have investigated the role of initial microstructure on hydrogen diffusion associated with embrittlement in pipeline steels [7]. Different grain sizes lead to different grain boundary surface areas per unit volume $\left(\mathrm{S}_{\mathrm{V}}\right)$. Therefore, to investigate the effect of this microstructural feature on hydrogen diffusion, three microstructures with different grain sizes, were used in the current CA model.

Previous work has shown that average grain volume (or average grain diameter) is a significant parameter affecting or controlling hydrogen diffusion because grain boundaries play a twofold role regarding hydrogen diffusion in the microstructure [6]. Grain boundaries can act as trap sites that limit hydrogen diffusion and as "pipelines" that facilitate migration. In the present work it is assumed that a large numbers of hydrogen atoms can be trapped at grain boundaries in very fine grained microstructures and numerous hydrogen atoms cannot find appropriate paths (grain boundaries) to diffuse into the microstructure in very coarse 
microstructures. Therefore, there is an optimized grain size with a specific $\mathrm{S}_{\mathrm{V}}$ which results in the highest diffusion rate of hydrogen in the steel microstructure.

\section{Model}

To simulate the diffusion process throughout the microstructure, an explicit 2D CA model was implemented. A 2D space with the given properties should be occupied by the diffusing hydrogen atoms. The space dimensions and the time step are divided into equal increments of $\delta \mathrm{x}, \delta \mathrm{y}$ and $\delta \mathrm{t}$, respectively. So the $\mathrm{X}-\mathrm{Y}$ coordination in each time step is covered by a grid of rectangles as shown in Fig. 1.

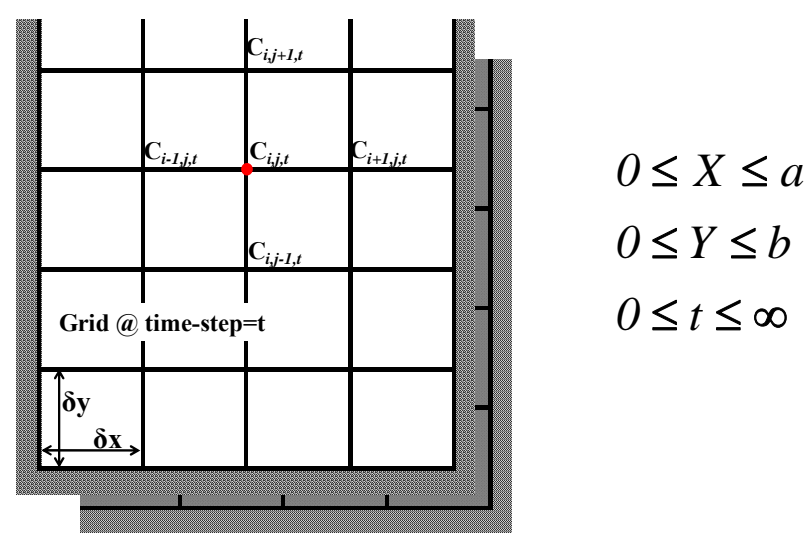

Figure 1: The coordination system and the grid size used in the model.

Let the coordinates of a representative grid point $(X, Y, T)$ be $(i \delta x, j \delta y, n \delta t)$, where $i, j, n$

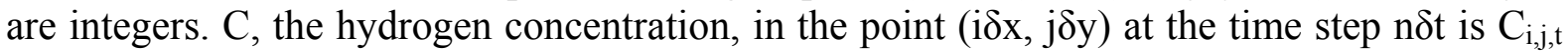
with corresponding values at the defined neighbouring points as in Fig. 1. The typical 2D diffusion problem, known as the second Fick's law, is expressed as [8]:

$$
\left(\frac{\partial c}{\partial T}\right)=D\left(\frac{\partial^{2} c}{\partial x^{2}}+\frac{\partial^{2} c}{\partial^{2} y}\right)
$$

where $\mathrm{c}$ is known as $\mathrm{c}_{0}$ over the whole grid (microstructure) at $\mathrm{T}=0 \mathrm{~s}$ and is prescribed on the boundaries for $\mathrm{T} \geq 0$. Therefore, the hydrogen concentration at each grid point is expressed by:

$$
c(i \delta x, j \delta y, n \delta t)=c_{i, j, n}
$$

Considering the Von-Neumann neighbourhood in the CA model, the finite difference scheme of Eq. 2 becomes:

$$
c_{i, j, n+1}=c_{i, j, n}+\frac{D \delta t}{(\delta x)^{2}}\left(c_{i-1, j, n}-2 c_{i, j, n}+c_{i+1, j, n}\right)+\frac{D \delta t}{(\delta y)^{2}}\left(c_{i, j-1, n}-2 c_{i, j, n}+c_{i, j+1, n}\right)
$$

Given the hydrogen concentration on the same grid point in the previous time step and given the hydrogen concentration on the neighbouring points at the same time step, the hydrogen concentration can be calculated using Eq. 3 .

\section{CA Model Set up}

In conventional CA, cells are equidistantly distributed over the microstructure [5] set for the progress of the diffusion front. As the input data to the CA model, an initial grid 
(microstructure) of $4000 \times 5000$ cells with the prescribed hydrogen concentration at the boundaries was employed to reduce the running time and the memory space usage. Calculation of the hydrogen concentration on each grid per time step considering the neighbouring cells is the main transition rule of the CA model.

\section{CA Neighbourhood}

To update the state (hydrogen concentration) of each cell, it is essential to know the state of the neighbouring cells in the current and in the previous time steps and it is also necessary to know the state of the considered cell in the previous time step. This spatial grid in which a cell needs to be considered is termed a neighbourhood area. According to the CA principles, theoretically there is no limitation in the neighbourhood size. However, it often includes only adjacent cells. In 2D CA application, three types of neighbourhoods (Fig. 2) (Von-Neumann, Moore and Octagon) are mostly used $[9,10]$. In Von-Neumann neighbourhood definition, which is implemented in the current model, the neighbourhood consists of a central cell (the one which has to be updated) and its four closest geographical neighbours [9,11] (Fig. 2a).

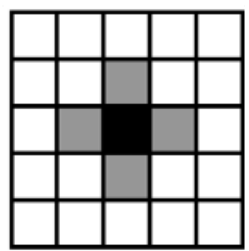

(a)

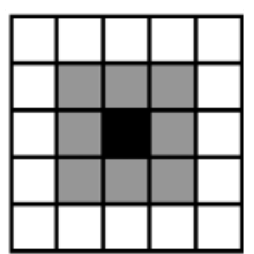

(b)
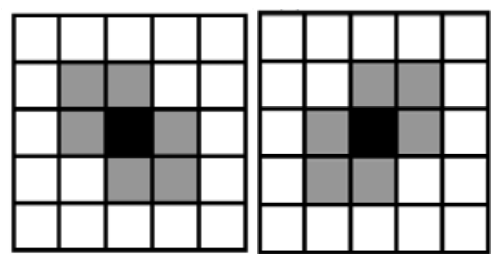

(c)

Figure 2: Three types of conventional neighbourhood in CA model. Von-Neumann (a), Moore (b) and Octagon (c). Black is the considered cell, grey cells are the neighbouring cells.

The grain boundaries in 2D were defined as the interface line between two differently oriented grains. To generate different microstructures by the CA model with different grain size, a given number of cells equal to the total number of grains were selected with the random coordination in the blank grid. Finally the neighbouring cells of each selected cell grew to obtain a fully impinged microstructure. As shown in Fig. 3, different microstructures with various grain sizes were generated and used in the model as the initial microstructures.

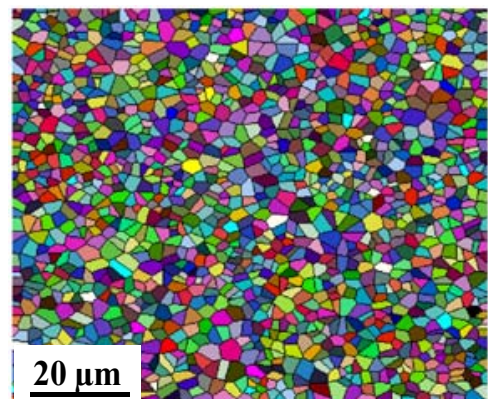

Total number of grains $=1547$ Average Grain Size $=5.7 \pm 0.05 \mu \mathrm{m}$ (a)

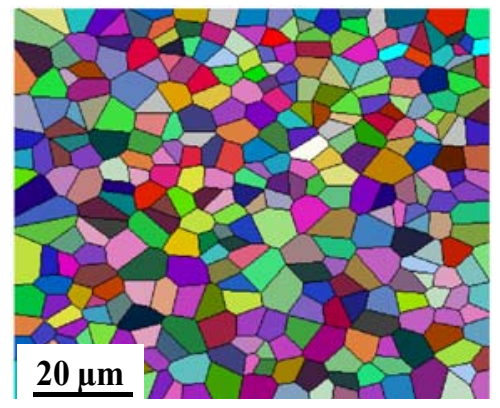

Total number of grains $=332$ Average Grain Size $=12.2 \pm 0.05 \mu \mathrm{m}$ (b)

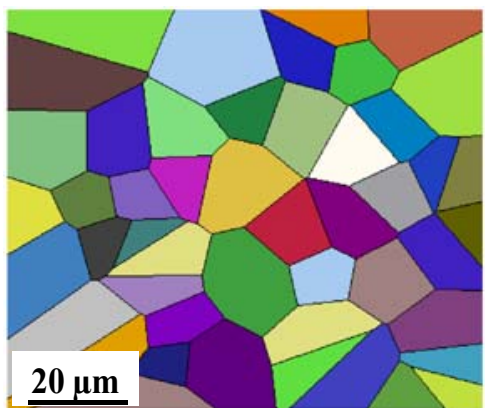

Total number of grains $=48$ Average Grain Size $=25.1 \pm 0.05 \mu \mathrm{m}$ (c)

Figure 3: The initial microstructures with different grain sizes generated and used in the CA model.

\section{Assumptions of the CA Model}

In the generated microstructures, grains and the associated cells were distinguished from each other by their different orientations and by their level of hydrogen concentrations. The microstructures generated by the CA model are assumed to be part of the pipeline body. As shown in Fig. 4, there is continuous flow of hydrogen gas in the pipeline. Therefore, the 
internal hydrogen pressure (hydrogen concentration on the internal surface of the pipeline) remains constant during the entire simulation and the outer surface of the pipe is in contact with air as for real in-service pipelines. The service temperature is also supposed to be constant.

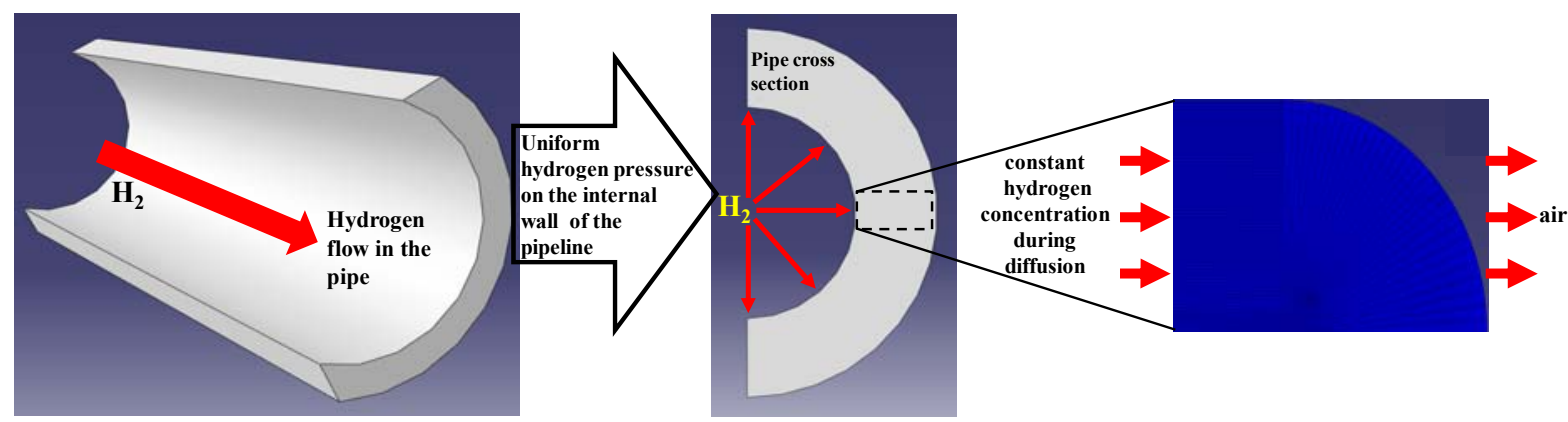

Figure 4: The assumptions for the diffusion boundary conditions of the microstructure.

\section{Simulation Stages}

Modelling hydrogen diffusion using the CA model consisted of the following stages:

1- Since, X70 pipeline steel is used in experiments, the associated constants of X70 steel are utilized in the model [12].

2- Considering a part of the pipe body, the left side of the microstructure which is the internal surface of the pipe body has higher hydrogen concentration than the right side during the entire simulation process (Fig. 4).

3- Unlike other models in which diffusion stops when a steady state is reached, in the current model the diffusion process continues beyond the steady state and the overall concentration of hydrogen in the microstructure increases to achieve the same concentration as that of the internal surface of the pipe.

4- The boundary conditions are applied and the hydrogen concentration of each grid is calculated using Eq. 3.

\section{Results and Discussion}

As shown in Fig. 5, $\mathrm{X}$ and $\mathrm{Y}$ are the coordinates of the $\mathrm{CA}$ grid on the microstructure and $\mathrm{Z}$ represents the hydrogen concentration value at each grid point. The figure demonstrates the diffusion front which moves through the microstructure from the left side to the right side. To save running time and to study the effect of grain size on hydrogen diffusion a microstructure with three average grain sizes is considered (layers of different grain sizes are separated by dashed lines in the schematic microstructure shown in Fig. 5a.

There is a considerable gradient of hydrogen concentration when diffusion starts (Figs. $5 \mathrm{~b}$ and c). As shown in the initial steps of simulation, the diffusion front moves forward at different rates depending on the average grain size. However, as diffusion progresses the concentration gradients among different grain sizes are diminished (Figs. 5 d). The gradient disappears because the hydrogen concentration in each cell at each time step was calculated considering the concentration in the neighbouring cells. Since all grain boundaries are supposed to have the same diffusion behaviour, there is no priority in receiving hydrogen atoms from the sender cell of the similar grain or from the sender cell of a dissimilar grain and the hydrogen concentration gradient will be the only driving force for diffusion. Hence, regardless of which grain the cell belongs to, the larger the time step the lower the gradient will be among the cells (Fig. $5 \mathrm{c}$ ). 


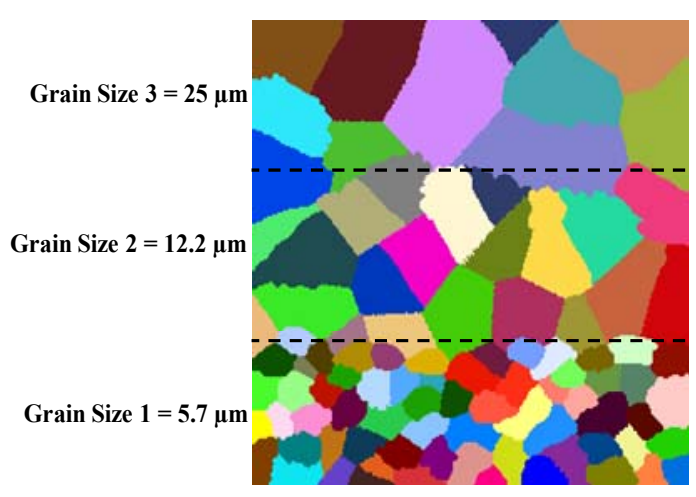

(a)

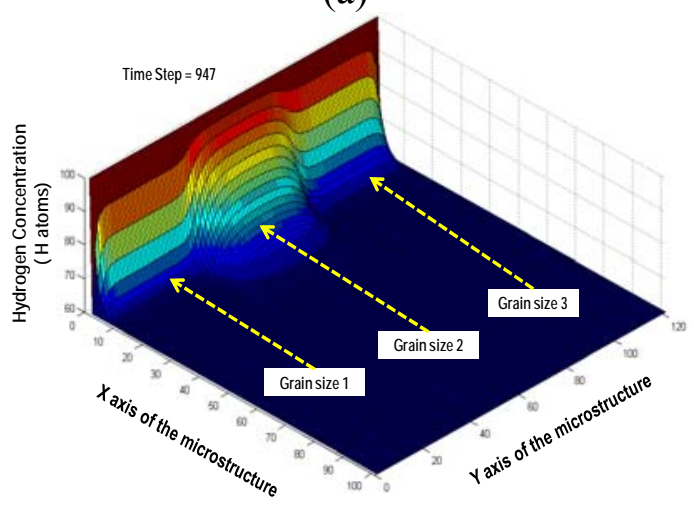

(c)

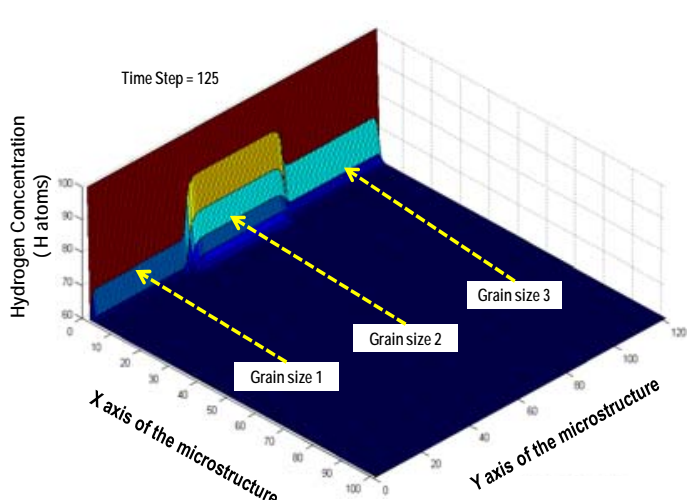

(b)

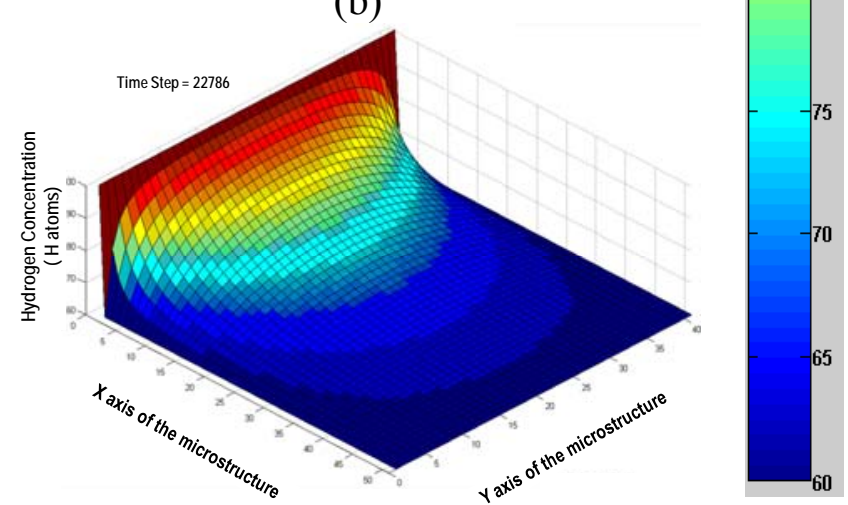

(d)

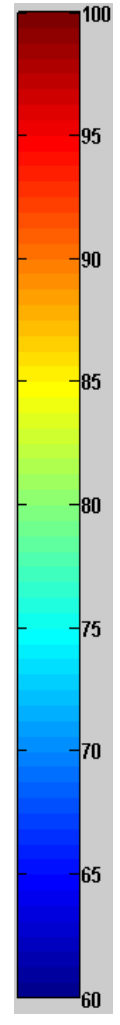

Figure 5: A 3D demonstration of the progress of hydrogen diffusion through microstructures shown schematically as grain sizes 1,2 and 3 (a), after the time intervals indicated in (b) to (d). The left side in figures (b) to (d) has the highest level of hydrogen and the right side is in contact with air. Grain Size $1=$ 5.7, Grain Size $2=12.2$ and Grain Size 3= $25 \mu \mathrm{m}$.

Grain boundaries have a two-fold effect on diffusion [13]. From one side there is an enhancement of hydrogen diffusion along grain boundaries, whereas there can also be a hydrogen trapping effect by the boundary network [13]. As the grain size decreases, $\mathrm{S}_{\mathrm{V}}$ increases and the hydrogen mobility will increase as a function of increasing grain boundary area. However, a fine-grained microstructure has a much higher density of triple junctions as potential traps for hydrogen atoms, which leads to the reduction of hydrogen mobility. As a result of these two contradictory effects, the hydrogen diffusion coefficient will be maximised for a specific grain size (Fig. 6). This effect of grain boundaries on the hydrogen diffusivity is called the "grain boundary cross effect" [13].

The simulation results of diffusion in the three microstructures with different grain sizes show that there is an optimal grain size that results in the highest hydrogen diffusivity. As shown in Fig. 6, the simulation results for steel in the present case and the experimental results for aluminum [13] show the same trend, with a maximum diffusion coefficient occurring at an intermediate grain size. The simulation results indicate that diffusion along grain boundaries can play an important role in the overall diffusion process. 


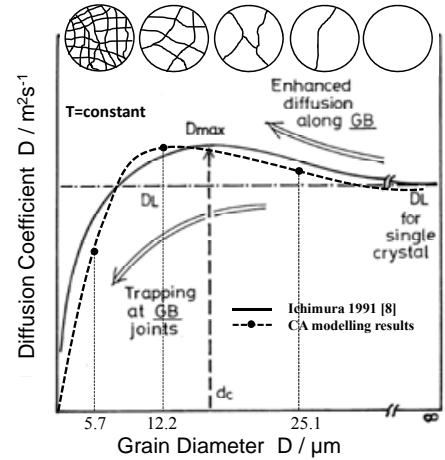

(a)

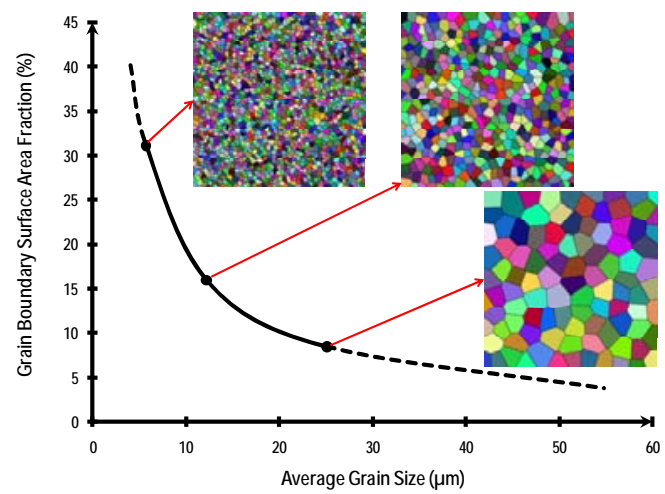

(b)

Figure 6: The effect of average grain size on the diffusion coefficient [12] (a), the relation between average grain size and the surface area fraction.

\section{Conclusion}

The average grain size and, more particularly, the average grain boundary surface area / per unit volume can play a significant role in the diffusion process. The results also support the hypothesis that grain boundaries can exert opposing effects on the diffusivity of hydrogen and that the most rapid diffusion is expected for an intermediate grain size.

\section{Acknowledgment}

This work was conducted as part of an Australian Research Council (ARC) Linkage Grant LP0883546 with BlueScope Steel as Industrial Partner. We gratefully acknowledge the financial support of the ARC and Bluescope Steel.

\section{References:}

1. O. L. Bandman, Parallel Computing Technologies, 1662, (1999), 395-409.

2. B. Chopardt, M. Drozt, M. KolbS, Cellular automata approach to non-equilibrium diffusion and gradient percolation, J. Phys. A: Math. Gen. 22, (1989), 1609-1619.

3. G.Doolen, Addison-Wesley, 1990

4. B. Chopard, M. Droz and M. Kolb, Journal of Physics, A22, (1989), 1609.

5. B. Chopard and M. Droz, Journal of Physics, A21, (1988), 205.

6. D. Dab and J. P. Boon, P. Manneville, ed. (Springer-Verlag), (1989), 257.

7. B. Chopard, M. Droz, Cellular Automata Model for the Diffusion Equation, Journal of Statistical Physics, 64 (3-4), (1991), 859-892.

8. J. Crank, The Mathematics of Diffusion, Second Edition, Oxford Press, (1975), 24.

9. H. W. Hesselbarth, I. R. Gobel, Simulation of Recrystallization by Cellular Automata. Acta Metall. Mater., 39 (9), (1991), 2135-2143.

10. C. H. J. Davies, The effect of neighbourhood on the kinetics of a cellular automaton recrystallisation model, Scripta Metal. et Mater., 33, (1995), 1139-1143.

11. H. W. Hesselbarth, L. Kaps, F. Hassner, Two Dimensional Simulation of the Recrystallization Kinetics in the Case of Homogeneously Stored Energy, Material Science Forum, 113, (1993), 317 - 322.

12. M. Dadfarnia, P. Sofronis, I. Robertson, B. P. Somerday, G. Muralidharan, D. Stalheim, "Numerical Simulation of Hydrogen Transport at a Crack Tip in a Pipeline Steel,"submitted for the proceedings of IPC2006, 6thInternational Pipeline Conference, (2006) 25-26.

13. M. Ichimura, Y.Sasajima, M. Imabayashi, Grain boundary effect on diffusion of hydrogen in pure Aluminum, Materials Transactions, 32(12), (1991), 1109-1114. 